\title{
An Efficient Technique to Detect Visual Defects in Particleboards
}

\author{
Jonas GUZAITIS \\ Department of Applied Electronics, Kaunas University of Technology, \\ LT-51368, Kaunas, Lithuania \\ e-mail: jonas.guzaitis@ktu.lt \\ Antanas VERIKAS \\ Department of Applied Electronics, Kaunas University of Technology, \\ Intelligent Systems Laboratory, Halmstad University \\ Box 823, S-30118 Halmstad, Sweden \\ e-mail: antanas.verikas@hh.se
}

Received: 26 March 2007; accepted: 3 June 2008

\begin{abstract}
This paper is concerned with the problem of image analysis based detection of local defects embedded in particleboard surfaces. Though simple, but efficient technique developed is based on the analysis of the discrete probability distribution of the image intensity values and the 2D discrete Walsh transform. Robust global features characterizing a surface texture are extracted and then analyzed by a pattern classifier. The classifier not only assigns the pattern into the quality or detective class, but also provides the certainty value attributed to the decision. A $100 \%$ correct classification accuracy was obtained when testing the technique proposed on a set of 200 images.
\end{abstract}

Keywords: defect detection, image analysis, Walsh transform.

\section{Introduction}

Particleboards are made of pressed wood particles and a suitable binder. Bigger particles compound the inner part of the material and smaller ones - the outer surface. Such structure makes particleboards strong and smooth. During the production process, defects may appear on a board's surface. The most common defects in particleboards are hollows in a surface near the border as shown in Fig. 1 (top-right). Such defects may occur during the cutting phase or due to bad trimming after compression. Other types of defects are various blots. Two examples of such blots are shown in Fig. 1 (bottom).

To increase the competitiveness of the products, defective particleboards must be detected and prevented from being sent to a customer. Thus industrial quality inspection is required. Image analysis and soft computing techniques are being increasingly used to automate industrial inspection in various fields and for surveillance as well (Kisel et al., 2008; Ghita et al., 2005; Verikas et al., 2003; Kumar, 2003; Verikas et al., 2005; Bacauskiene and Verikas, 2004; Ribaric et al., 2008; Verikas et al., 2000). Most of the available image analysis based defect detection systems focus on non-textured surfaces such as 

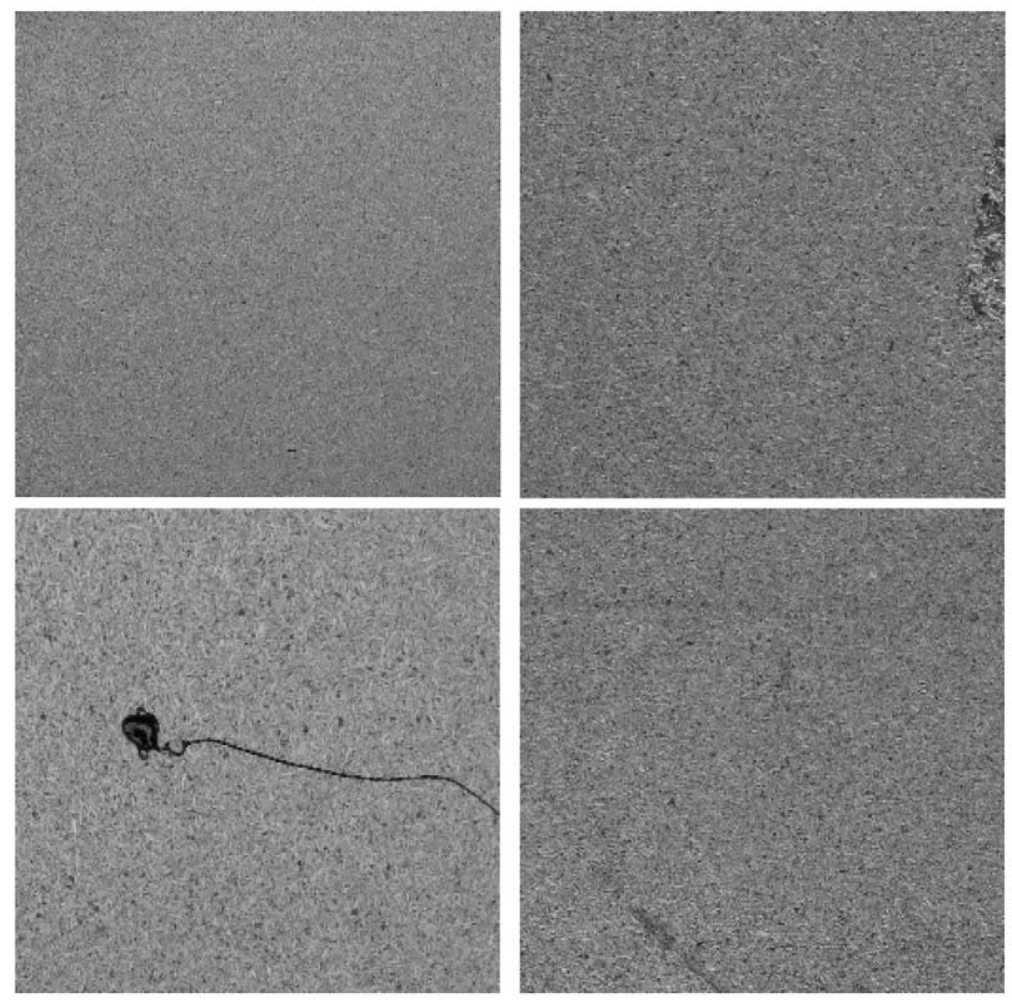

Fig. 1. Examples of particleboard surfaces: (top-left) quality surface, (top-right) hollow near the border, (bottom-left) pool of oil, (bottom-right) blot.

glass panel (Ghita et al., 2005; Tsai and Lin, 2002), sheet steel (Pernkopf, 2004), and textile materials (Kumar, 2003; Bodnarova et al., 2000; Nagan et al., 2005; Tsai and Hsieh, 1999). Defects in such images are rather easily detected because they manifest themselves by distinctly measured values if compared to those of the uniform background.

A spotty surface of a particleboard generates a textured image, see Fig. 1 (top-left). Techniques for automatic visual inspection of textured images generally compute a set of textural features in the spatial or spectral domain and then search for significant local deviations of the feature values if compared to those computed using defect free images. The first and second-order statistics derived from the gray-level co-occurrence matrices are the commonly used features in the spatial-domain (Latif-Amet et al., 2000). However, using this technique it is difficult to locate the defect position. Another popular approach is based on image filtering and simple thresholding. Fourier transform (Tsai and Huang, 2003; Chan and Pang, 2000), Gabor transform (Tsai and Lin, 2002) or Wavelet transform (Nagan et al., 2005; Tsai and Huang, 2003) is usually applied before the thresholding. However, since multiple filters are usually used, the image filtering phase is rather time consuming. Large computation time characteristic to sophisticated techniques prevents them from being implemented in industry for online detection of visual defects. 
Nonetheless the significant progress in automated image analysis based industrial visual inspection, currently, the inspection process in particleboard production still depends mainly on human sight. The nature of this work is very dull and repetitive. Moreover, there could be many human errors in this process. According to some studies, due to tiredness and human errors, human visual inspection can only catch around $60-75 \%$ of the significant visual defects (Schicktanz, 1993).

In this study, we present a simple, but efficient technique for surface defect detection in particleboards. A low computation time and a high defect detection reliability are two characteristic features the technique is aiming at. A textured image recorded from a particleboard surface is first subjected to the global histogram based analysis. Dark large scale blobs are already detected in this stage of the analysis. To detect other defects, features characterizing the surface texture are extracted and analyzed by a pattern classifier designed to categorize feature vectors into the quality and defective classes. Features characterizing the shape and position of the image intensity histogram and the coefficients of the 2D discrete Walsh transform constitute the feature vector. Some visual defects can be effectively detected by analyzing the frequency content of an image. The 2D discrete Walsh transform is a very simple tool to represent an image in a frequency domain. Therefore, the Walsh transform has been utilized in this study. Along with the decision regarding the class of the surface being analyzed, the certainty of the decision is also provided. In the following sections the technique proposed is thoroughly described. All the experimentally chosen parameters the analysis results depend on are discussed at the beginning of Section 4.

\section{The Approach}

\subsection{Image Acquisition}

Surface hollows are not distinguishable from the background if the light source used is diffusive or directed in parallel to the camera view. This is because the defective and background areas are of the same material and just relief is different. To solve the problem, we use spotlight directed to the surface almost in parallel to the board. When using such illumination, edges of the hollows make shadows, which are much easer to detect.

To cope with the problem of uneven illumination, an average image computed from a large number of defect-free surfaces is subtracted from an image being analyzed. Having an image $f$, the normalized image $f_{n}$ is given by

$$
\mathbf{f}_{n}=\mathbf{f}-\overline{\mathbf{f}}+\mathbf{m}
$$

where $\overline{\mathbf{f}}$ is the average image and $\mathbf{m}$ is an image with gray values of all pixels equal to the mean gray of $\overline{\mathrm{f}}$. 


\subsection{Feature Extraction}

Two information sources are used to extract features in this study, namely, the discrete probability distribution given by the histogram of the image intensity values and the coefficients of the 2D discrete Walsh transform. Some defects like large dark blobs can be easily detected by simple image thresholding, for example histogram based. Fig. 2 presents two examples of images taken from defective particleboard surfaces. It is obvious that the defect seen in the left-hand-side image can be easily detected by simple thresholding applied to the low-pass filtered image. However, this is not the case with the image shown on the right. The gray value histograms of the images shown in Fig. 3 substantiate the fact.

To find the optimal threshold value $t^{*}$ for accomplishing the thresholding, we use the histogram based technique (Otsu, 1979).

Let $L$ be the number of grey levels in the image and $K_{i}$ the number of pixels at a grey level $i$. The total number of pixels in a given image is then equal to $K=\sum_{i=1}^{L} K_{i}$ and the probability of a grey level $i$ is defined as $p_{i}=K_{i} / K$. The histogram of the image is then given by $h(i)=K_{i}$. The optimal $t^{*}$ sought is such that the between-cluster variance $\sigma_{B}^{2}(t)$ is maximized when dividing the histogram into two clusters $C_{0}$ and $C_{1}$ at
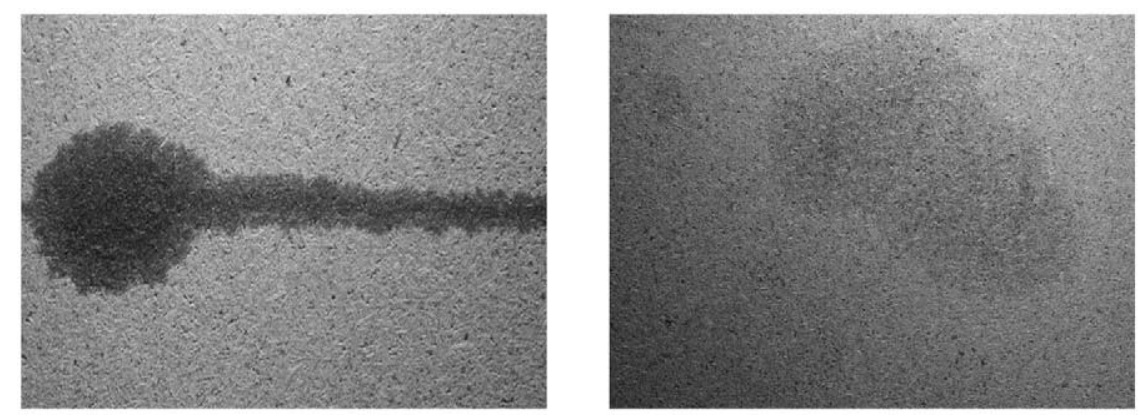

Fig. 2. Examples of two images taken from defective particleboard surfaces.
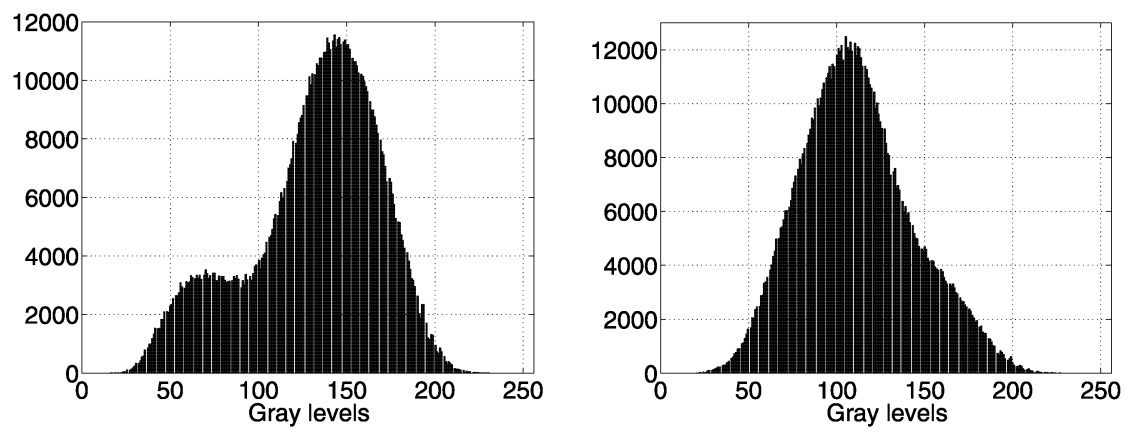

Fig. 3. Intensity histograms of the images shown in Fig. 2. 
the image intensity (gray) value equal to $t^{*}$ :

$$
\sigma_{B}^{2}\left(t^{*}\right)=\max _{t \in T} \sigma_{B}^{2}(t)
$$

where $T$ is a set of the gray values restricted by the boundary values of the histogram. The between-cluster variance is given by

$$
\sigma_{B}^{2}(t)=P_{0}\left(\mu_{0}-\mu_{T}\right)^{2}+P_{1}\left(\mu_{1}-\mu_{T}\right)^{2}
$$

where $P_{0}$ and $P_{1}$ stand for the cluster occurrence probabilities, $\mu_{0}$ and $\mu_{1}$ are means of the clusters, and $\mu_{T}$ is the total mean. A high $\sigma_{B}^{2}\left(t^{*}\right)$ value (exceeding some predetermined threshold) indicates the fact of reliable defect detection via the histogram based image thresholding. We defined the following measure to assess the reliability of the thresholding result:

$$
\gamma_{T}(\mathbf{f})=1-\exp \left\{-\alpha_{T} \sigma_{B}^{2}\left(t^{*}\right)\right\}
$$

where the experimentally chosen parameter $\alpha_{T}$ determines the sensitivity of the measure. The measure ranges between 0 and 1 .

If for a given image $\mathbf{f}(x, y)$ the reliability of the thresholding step is below a predetermined value $\left(\gamma_{T}(\mathbf{f})<\delta_{T}\right)$, features characterizing the histogram shape and the coefficients of the 2D Walsh transform are extracted for further analysis.

\subsubsection{Characterizing Histogram}

Fig. 4 presents two histograms computed using the defect free surface (left) and the surface contaminated by a small hollow. As it can be seen from Fig. 4, the histogram calculated using the defective surface has a longer tail. This is a characteristic feature of histograms obtained from defective surfaces.

We use several features to characterize a histogram, namely:

- mean value $m$,

- standard deviation $\sigma$,
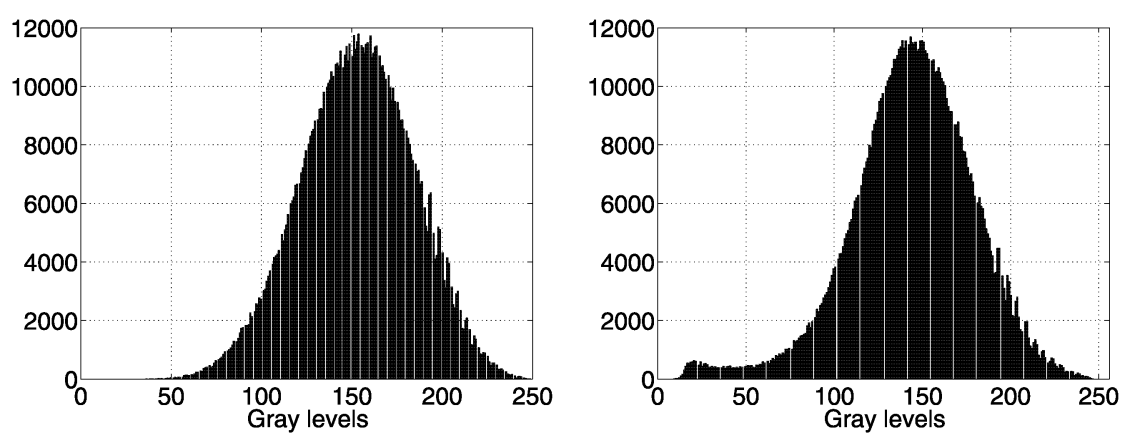

Fig. 4. Intensity histograms of images obtained from the quality (left) and defective (right) surfaces. 
- skewness $\mu_{3}$,

- kurtosis $\mu_{4}$,

- percentile 5 value,

- percentile 95 value,

- $\sigma_{B}^{2}\left(t^{*}\right)$ value.

The standard deviation is given by

$$
\sigma=\sqrt{\frac{1}{K-1} \sum_{i=1}^{L}(i-m)^{2} h(i)}
$$

where $m=\frac{1}{K} \sum_{i=1}^{L} h(i) i$ is the mean value and $K$ is the total number of pixels in a given image.

Skewness $\mu_{3}$ is a measure of the asymmetry degree of a histogram around the mean value. The more asymmetric is the histogram, the larger is the skewness value. A histogram skewed to the left possesses a negative $\mu_{3}$ value, while a positive $\mu_{3}$ value is computed for a histogram skewed to the right (Theodoridis and Koutroumbas, 2003). Skewness is given by

$$
\mu_{3}=\sigma^{-3} \sum_{i=1}^{L}(i-m)^{3} h(i)
$$

Kurtosis (excess kurtosis) is defined as

$$
\mu_{4}=\sigma^{-4} \sum_{i=1}^{L}(i-m)^{4} h(i)-3
$$

The normal distribution has kurtosis $\mu_{4}=0$. Positive kurtosis is a sign of a "peaked" distribution while negative kurtosis indicates a "flat" distribution. We expect that defective surfaces produce non-symmetrical histograms with non-zero skewness and large kurtosis values. By large kurtosis values we mean values resulting in the average kurtosis value that is statistically significantly different from the average kurtosis value computed for defect-free surfaces.

\subsubsection{The 2D Discrete Walsh Transform Based Analysis}

The 2D discrete Walsh transform (Gonzalez and Woods, 2002; Petrou et al., 1996) is a simple tool for representing an image in a frequency domain. The Walsh functions constitute a system of orthogonal functions acquiring values equal to only \pm 1 . The Walsh functions can be generated using the Hadamard matrix $\mathbf{H}$. The Hadamard matrices can be defined recursively:

$$
\mathbf{H}_{2}=\left[\begin{array}{ll}
+1 & +1 \\
+1 & -1
\end{array}\right]
$$




$$
\mathbf{H}_{4}=\mathbf{H}_{2} \otimes \mathbf{H}_{2}=\left[\begin{array}{ll}
+\mathbf{H}_{2} & +\mathbf{H}_{2} \\
+\mathbf{H}_{2} & -\mathbf{H}_{2}
\end{array}\right]=\left[\begin{array}{llll}
+1 & +1 & +1 & +1 \\
+1 & -1 & +1 & -1 \\
+1 & +1 & -1 & -1 \\
+1 & -1 & -1 & +1
\end{array}\right],
$$

where $\otimes$ stands for the Kroneker's product. Similarly, a $2^{k} \times 2^{k}$ Hadamard matrix can be obtained.

Given an image $\mathbf{f}(x, y)$ of $N \times N$ pixels, where $N$ is a power of 2 , the $2 \mathrm{D}$ discrete Walsh transform $\mathbf{W}$ of the image is defined as

$$
\mathbf{W}=\frac{1}{N} \mathbf{H}_{N} \mathbf{f} \mathbf{H}_{N}
$$

Fig. 5 (left) presents an image taken from a particleboard surface and its corresponding 2D Walsh transform (middle). The lowest frequencies are placed at the top-left corner.

To be able to utilize frequencies $(u, v)$ from only some frequency region $R$ in the feature extraction process, a frequency mask $\nu(u, v)_{R}$ is defined:

$$
\nu(u, v)_{R}= \begin{cases}1, & \text { if } u, v \in R \\ 0, & \text { if } u, v \notin R\end{cases}
$$

The region of low frequencies $R_{L}$ used to extract features for defect detection is delimited by an arc drawn at the chosen frequency $f_{L}$. Thus,

$$
\nu(u, v)_{L}= \begin{cases}1, & \text { if } 0<\sqrt{u^{2}+v^{2}}<f_{L}, \\ 0, & \text { otherwise. }\end{cases}
$$

The mask used to extract features for defect detection is illustrated in Fig. 5 (right). Observe that we set $\nu(0,0)=0$. Then, having the filtered 2D Walsh transform image, the Walsh transform based features are given by the following parameters:

$$
m_{W}=\frac{1}{\operatorname{card}\left\{R_{L}\right\}} \sum_{u, v \in R_{L}}\left|W_{u, v}\right|,
$$
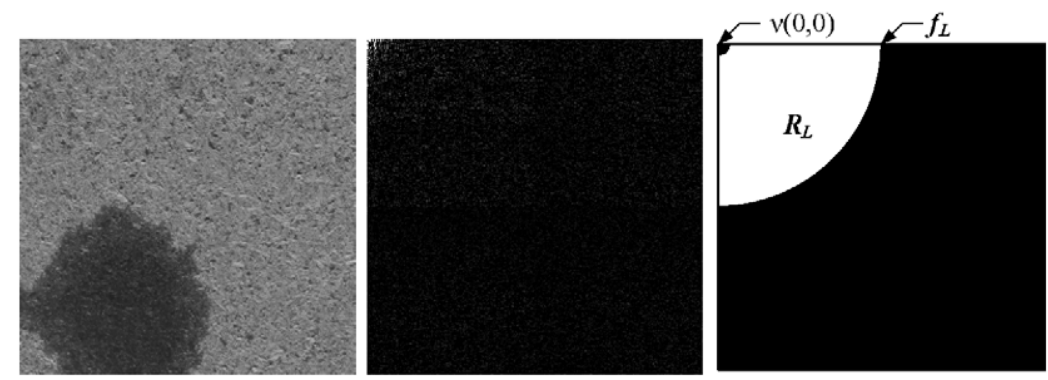

Fig. 5. An image (left), its corresponding 2D Walsh transform (middle), and the frequency mask (right). 


$$
s_{W}=\sqrt{\frac{1}{\operatorname{card}\left\{R_{L}\right\}-1} \sum_{u, v \in R_{L}}\left(\left|W_{u, v}\right|-m_{W}\right)^{2}},
$$

where $W_{u, v}$ stands for the coefficients of the transform and card $\left\{R_{L}\right\}$ is the cardinality of the set $R_{L}$. The parameters $m_{W}$ and $s_{W}$ express the average magnitude of the Walsh transform coefficients and the standard deviation of the magnitude, respectively. Images taken from even surfaces exhibit lower values of $m_{W}$ than from uneven ones.

\subsection{Decision Making}

Extracted features are collected into a vector $\mathbf{z}$ and used by a classifier to make a decision about the particleboard class the image was recorded from - defective or quality. The feature vector $z$ consists of nine components: the seven features characterizing a histogram and the parameters $m_{W}$ and $s_{W}$. The best feature subset, the one providing the highest classification accuracy, was found experimentally, by applying the sequential forward feature selection procedure. Two types of classifiers have been used in this study, the linear discriminant function and the multilayer perceptron.

Let $\mathbf{z}$ be a feature vector. The discriminant function $g(\mathbf{z})$ is then given by

$$
g(\mathbf{z})=w_{0} z_{0}+w_{1} z_{1}+w_{2} z_{2}+\cdots+w_{n} z_{n}
$$

where $z_{0}=1, n$ stands for the number of features used and $\mathbf{w}$ denotes a parameter vector. A given pattern characterized by a feature vector $\mathbf{z}$ is assigned to the quality class if $g(\mathbf{z})>0$ and the defective class otherwise. The optimal values of the parameter vector $\mathbf{w}$ are found by solving a system of linear equations.

A feed-forward multilayer perceptron with one hidden layer and the hyperbolic tangent transfer functions in both the hidden and the output layers has been used. The optimal number of the hidden nodes has been found experimentally. Cross-validation data set based early stopping is usual way to control overfitting. However, due to the relatively small data sets available, we avoided using cross-validation data sets. To avoid overfitting, we used Bayesian regularization (MacKay, 1992; Bishop, 1995), which is implemented by minimizing the following objective function:

$$
E=\frac{\beta}{2} \sum_{i=1}^{N_{D}} \sum_{j=1}^{Q}\left\{y_{j}^{i}\left(\mathbf{z}_{i} ; \mathbf{w}\right)-t_{j}^{i}\right\}^{2}+\frac{\alpha}{2} \sum_{s=1}^{N_{W}}\left(w_{s}\right)^{2}
$$

where $\mathbf{z}_{i}$ is the input data point, $N_{D}$ is the number of training data points, $Q$ is the number of outputs in the network (one in our case), w is the weight vector, $N_{W}$ is the number of weights, $y_{j}^{i}\left(\mathbf{z}_{i} ; \mathbf{w}\right)$ is the output value of the $j$ th output node, $t_{j}^{i}$ is the target value for the $j$ th output node given the input pattern $\mathbf{z}_{i}$, and $\alpha$ and $\beta$ are the hyper parameters.

The second term of the objective function performs regularization. In the Bayesian approach, the weights of the network are considered as random variables and the optimal values of the hyper-parameters $\alpha$ and $\beta$ are found automatically in the learning process. 


\subsection{Assessing the Reliability of the Analysis}

The analysis results obtained from the techniques are evaluated by calculating values of reliability measures. Values of the measures give an indication to which extent we can trust the analysis results. The reliability measure used to asses the thresholding result has already been discussed and is given by (4).

A linear discriminant function $g(\mathbf{z})$ divides the feature space into two parts by a hyperplane. Given a pattern $\mathbf{z}$, the distance $d(\mathbf{z})$ of the pattern from the hyper-plane can be defined as

$$
d(\mathbf{z})=g(\mathbf{z}) /\|\mathbf{w}\|
$$

where $\|\cdot\|$ stands for the vector norm. We exploit this distance, to assess the classification reliability. The classification reliability measure $\gamma_{g}(\mathbf{z})$ is given by

$$
\gamma_{g}(\mathbf{z})=1-\exp \left\{-\alpha_{g}|g(\mathbf{z})|\right\}
$$

where $\alpha_{g}$ is a parameter chosen experimentally. The $\gamma_{g}(\mathbf{z})$ measure ranges between 0 and 1 . The larger the value, the more reliable is the decision. For a feature vector $\mathbf{z}$ lying on the separating hyper-plane, $\gamma_{g}(\mathbf{z})=0$. In the neural network case, we directly used the network output value to assess the decision reliability.

In addition to the defective and quality classes a rejection class is also used in the decision making process. A given image $\mathbf{f}(x, y)$ taken from a particleboard surface is assigned to the rejection class if

$$
\gamma_{g}(\mathbf{f})<\delta_{g}
$$

with $\delta_{g}$ being a threshold chosen experimentally.

\section{Localizing Defects}

Defects can be detected in the histogram thresholding phase or in the second phase during the classification based on the histogram and Walsh transform features. Defects detected in the first phase are localized directly during the thresholding. Localization of defects detected during the second phase is based on the inverse Walsh transform of the filtered Walsh transform image.

First, a filtered Walsh transform image $\mathbf{W}_{f}$ is obtained

$$
\mathbf{W}_{f}=\mathbf{W} \cdot \nu
$$

where - stands for the element-wise multiplication and $\nu$ is the mask defined in (12). Next, a reconstructed image $\mathbf{f}_{r}$ is created using the inverse Walsh transform

$$
\mathbf{f}_{r}=\frac{1}{N} \mathbf{H}_{N} \mathbf{W}_{f} \mathbf{H}_{N}
$$



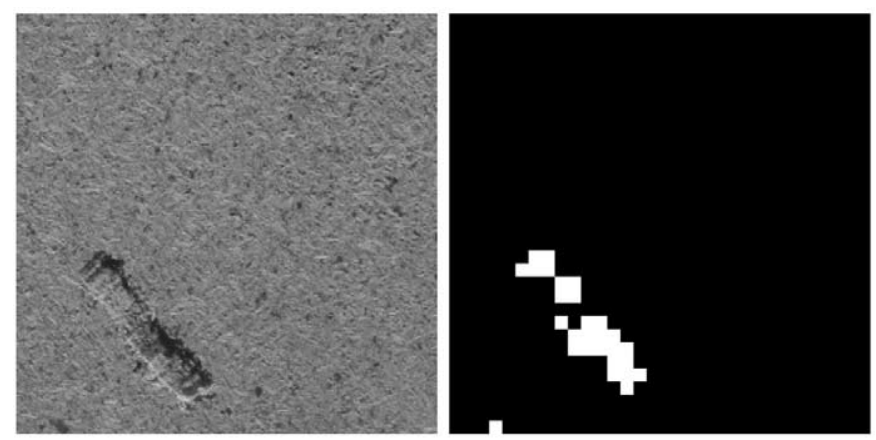

Fig. 6. An image of the defective surface (left) and the defect detection result (right).

The reconstructed image $\mathbf{f}_{r}$ is then subjected to the histogram-based thresholding discussed in Section 2.2. Fig. 6 presents an example of the defect localization result. As one can see, even the small hollow at the left-bottom corner has been detected. Defect shape angularity is a result of the Walsh transform specific constituents - orthogonal step functions.

\section{Experiments}

Images we used in the experiments were of $256 \times 256$ pixels size and $L=256$ gray levels. All the images were taken by a still photo camera. In total, 100 quality and 100 defective surfaces were available. Fig. 1, Fig. 2 and Fig. 5 present some examples of the images used. It is worth noting that the average intensity value of the images used in the experiments varied in a rather broad range. Estimation of the correct classification rate obtained from the technique proposed was carried out using the leave-one-out approach, meaning that all the available data points, $D$, were used for training, except one left aside, which was used for testing. This process is repeated $D$ times, each time leaving aside a different data point. Thus, the size of the training set used in the experiments is 199 .

Values of the parameters $f_{L}, \alpha_{g}, \alpha_{T}, \delta_{g}$, and $\delta_{T}$ have been chosen experimentally. The values of $\alpha_{g}=1, \alpha_{T}=10$, and $\delta_{T}=0.7$ worked well in all the tests. The value of $\delta_{g}$ depends on the cost of accepting a wrong decision. In this work, we set the value to $\delta_{g}=0.7$. The parameter $f_{L}$ affects the discrimination power of the Walsh transform based features. Fig. 7 illustrates the dependence of the feature $m_{W}$ on the parameter $f_{L}$ for the quality and defective classes. One hundred images from each class have been used to obtain the dependencies. Based on the experimental tests, we set $f_{L}=75$.

\subsection{Results}

First, we assessed the discrimination power of the features by calculating the Fisher index. The Fisher index of the $i$ th feature is given by

$$
J_{i}=\frac{\left(\mu_{i q}-\mu_{i d}\right)^{2}}{\sigma_{i q}^{2}+\sigma_{i d}^{2}}
$$




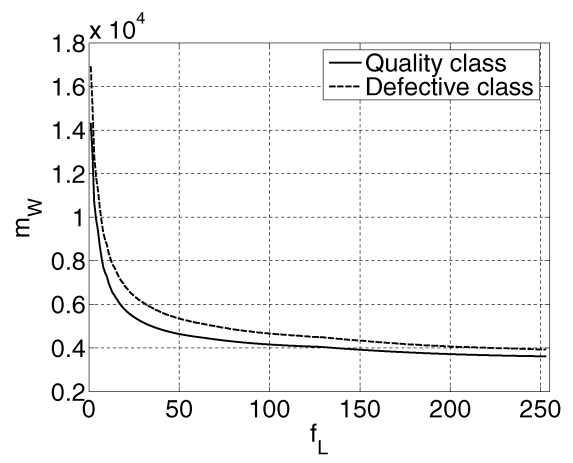

Fig. 7. The feature $m_{W}$ as a function of the parameter $f_{L}$ for the quality and defective classes.

with $\mu_{i q}, \sigma_{i q}^{2}, \mu_{i d}$, and $\sigma_{i d}^{2}$ being the mean value and the variance of the $i$ th feature for the quality and defective class, respectively. The index values of features are given in In Table 1. In Table 1, p5 and p95 denote the percentile 5 and percentile 95 feature, respectively. As it can be seen from Table 1, all the features exhibit rather low Fisher index vales. Features $m_{W}, \sigma$, and p5 are the most discriminative ones. However, the linear correlation between these three features is very high, see Table 2 . Therefore, one can expect that these three features are mutually redundant.

Table 1

Fisher index values of features used to detect defects

\begin{tabular}{lc}
\hline Feature & Criterion value \\
\hline$m$ & 0.0091 \\
$\sigma$ & 0.0178 \\
$\mu_{3}$ & 0.0083 \\
$\mu_{4}$ & 0.0077 \\
$\mathrm{p} 5$ & 0.0118 \\
$\mathrm{p} 95$ & 0.0013 \\
$\sigma_{B}^{2}\left(t^{*}\right)$ & 0.0051 \\
$m_{W}$ & 0.0204 \\
$s_{W}$ & 0.0091 \\
\hline
\end{tabular}

Table 2

Correlation coefficient values for the three most discriminative features

\begin{tabular}{lccc}
\hline & $\sigma$ & $\mathrm{p} 5$ & $m_{W}$ \\
\hline$\sigma$ & 1 & -0.954 & 0.955 \\
$\mathrm{p} 5$ & -0.954 & 1 & -0.892 \\
$m_{W}$ & 0.955 & -0.892 & 1 \\
\hline
\end{tabular}


Table 3

The correct classification rate $(\%)$ obtained from the multilayer perceptron

\begin{tabular}{lcc}
\hline Features & Quality surfaces & Defective surfaces \\
\hline$\sigma, m_{W}, \mathrm{p} 5$ & 100 & 100 \\
$m_{W}, \mathrm{p} 5$ & 99 & 81 \\
$\sigma, m_{W}$ & 98 & 81 \\
$\sigma, \mathrm{p} 5$ & 98 & 83 \\
All & 81 & 76 \\
\hline
\end{tabular}

Table 4

The correct classification rate $(\%)$ obtained from the linear discriminant function

\begin{tabular}{lcc}
\hline Features & Quality surfaces & Defective surfaces \\
\hline$\sigma, m_{W}, \mathrm{p} 5$ & 95 & 77 \\
$m_{W}, \mathrm{p} 5$ & 95 & 76 \\
$\sigma, m_{W}$ & 94 & 70 \\
$\sigma, \mathrm{p} 5$ & 93 & 76 \\
All & 95 & 75 \\
\hline
\end{tabular}

To find the best feature subset, the sequential forward feature selection procedure was employed. It was found, rather surprisingly, that the three most discriminative features constitute the best feature subset. In spite of the high correlation, all the three features are utilized. It means that in spite of the fact that the three features reflect approximately the same properties of images, there also some specific image properties captured by each of the features. All the attempts to remove or add any feature led to decrease of the correct classification rate. Table 3 summarizes the correct classification rate obtained for the large scale defects when using different feature subsets. The results presented are for the multilayer perceptron containing 3 nodes in the hidden layer. The number of the hidden nodes has been found experimentally. As it can be seen from Table 3, a perfect classification was obtained using the three very simple features.

The linear discriminant function provided a lower performance. The results obtained from the linear discriminant function are summarized in Table 4.

At the rather conservative reliability level chosen, $\delta_{g}=0.7$, four images were assigned to the rejection class. Fig. 8 illustrates the reliability of the decisions made. As it can be seen from Fig. 8 , most of the decisions are made with a very high reliability.

\section{Conclusions}

In this paper, the problem of detecting visual defects embedded in particleboard surfaces has been considered. Though simple, but very efficient technique has been presented. Defects like relatively large blobs are already detected in the histogram thresholding phase. If the histogram thresholding does not result into reliable defect detection, the second 

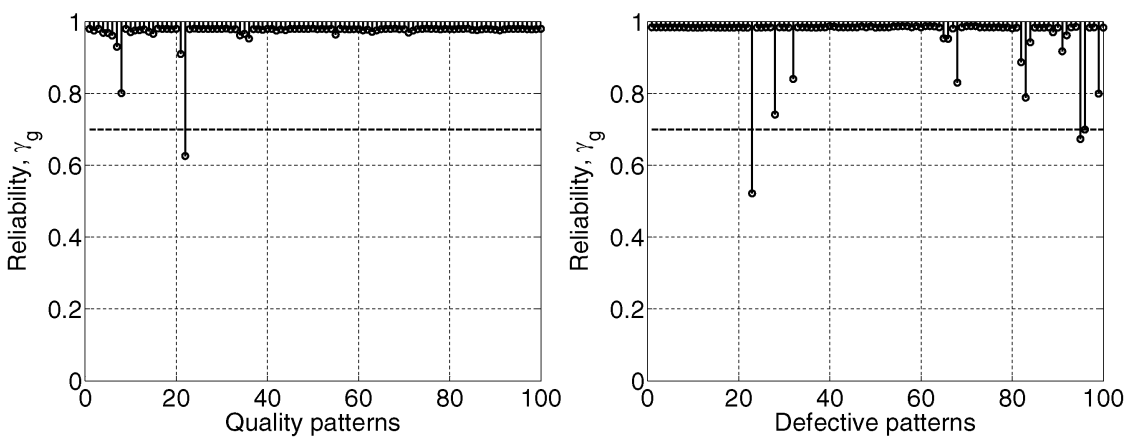

Fig. 8. The reliability $\gamma_{g}$ of the decisions made for the quality surfaces (left) and the defective ones (right) along with the reliability threshold $\delta_{g}=0.7$.

phase based on the analysis of the discrete probability distribution of the image intensity values and the $2 \mathrm{D}$ discrete Walsh transform is activated. The final decision is made by a classifier utilizing three very simple features characterizing the histogram shape and the average power of the Walsh transform coefficients. The location of the defects found is also estimated during the analysis. Two classifiers, namely a multilayer perceptron and a linear discriminant function, were tested. The multilayer perceptron trained with Bayesian regularization provided higher performance than the linear classifier. The rejection class inferred in the analysis allows avoiding decisions of low reliability. Surfaces leading to such decisions are allocated for posterior human inspection. A 100\% correct classification rate was obtained when testing the technique proposed on a set of $200 \mathrm{im}$ ages. Due to the type of features used, the detection results were rather insensitive to the quite large variation of the average intensity value of the images analyzed. The technique can be easily implemented in a simple hardware and used on production line.

\section{References}

Bacauskiene, M., and A. Verikas (2004). The evidence theory based post-processing of colour images. Informatica, 15, 315-328.

Bishop, C.M. (1995). Neural Networks for Pattern Recognition. Clarendon Press, Oxford.

Bodnarova, A., M. Bennamoun and K.K. Kubik (2000). Suitability analysis of techniques for flaw detection in textiles using texture analysis. Pattern Analysis \& Applications, 3, 254-266.

Chan, C.H., and K.H. Pang (2000). Fabric defect detection by Fourier analysis. IEEE Transactions on Industry Applications, 36, 1267-1276.

Ghita, O., P.F. Whelan, T. Carew and P. Nammalwar (2005). Quality grading of painted slates using texture analysis. Computers in Industry, 56, 802-815.

Gonzalez, R.C., and R.E. Woods (2002). Digital Image Processing. 2nd ed. Prentice Hall, Upper Saddle River, New Jersey.

Kisel, A., A. Kochetkov and J. Kranauskas (2008). Fingerprint minutiae matching without global alignment using local structures. Informatica, 19, 31-44.

Kumar, A. (2003). Neural network based detection of local textile defects. Pattern Recognition, 36, 1645-1659.

Latif-Amet, A., A. Ertuzun and A. Ercil (2000). An efficient method for texture defect detection: sub-band domain co-occurrence matrices. Image and Vision Computing, 18, 543-553.

MacKay, D.J. (1992). Bayesian interpolation. Neural Computation, 4, 415-447. 
Nagan, H.Y.T., G.K.H. Pang, S.P. Yung and M.K. Ng (2005). Wavelet based methods on patterned fabric defect detection. Pattern Recognition, 38, 559-576.

Otsu, N. (1979). A threshold selection method from gray-level histograms. IEEE Trans Systems Man \& Cybernetics, 9, 62-66.

Pernkopf, F. (2004). Detection of surface defects on raw steel blocks using Bayesian network classifiers. Pattern Analization \& Applications, 7, 333-342.

Petrou, M., J. Kittler and K.Y. Song (1996). Automatic surface crack detection on textured materials. Journal of Materials Processing Technology, 56, 158-167.

Ribaric, S., I. Fratric and K. Kis (2008). A novel biometric personal verification system based on the combination of palmprints and faces. Informatica, 19, 81-100.

Schicktanz, T. (1993). Automatic fault detection possibilities on nonwoven fabrics. Melliand Textilberichte, 74, 294-295.

Theodoridis, S., and K. Koutroumbas (2003). Pattern Recognition. 2nd ed. Academic Press, San Diego, CA.

Tsai, D.M., and C.P. Lin (2002). Defect detection on gold-plated surfaces on PCBs using entropy measures. Advanced Manufacturing Technology, 20, 420-428.

Tsai, D.M., and C.P. Lin (2002). Fast defect detection in textured surfaces using 1D Gabor filtres. Advanced Manufacturing Technology, 20, 664-675.

Tsai, D.M., and C.Y. Hsieh (1999). Automated surface inspection for directional textures. Image and Vision Computing, 18, 49-62.

Tsai, D.M., and T.Y. Huang (2003). Automated surface inspection for statistical textures. Image and Vision Computing, 21, 307-323.

Verikas, A., K. Malmqvist and L. Bergman (2005). Detecting and measuring rings in banknote images. Engineering Applications of Artificial Intelligence, 18, 363-371.

Verikas, A., M. Bacauskiene, A. Dosinas, V. Bartkevicius, A. Gelzinis, M. Vaitkunas and A. Lipnickas (2003). Intelligent system for tuning magnetic field of a cathode ray tube deflection yoke. Knowledge Based Systems, 16, 161-164.

Verikas, A., K. Malmqvist, M. Bacauskiene and L. Bergman (2000). Monitoring the de-inking process through neural network-based colour image analysis. Neural Computing \& Applications, 9, 142-151.

J. Guzaitis is a PhD student at the Department of Applied Electronics, Kaunas University of Technology. His research interests include image processing, pattern recognition, artificial neural networks, and applied soft computing.

A. Verikas is currently holding a professor position at both Halmstad University Sweden and Kaunas University of Technology, Lithuania. His research interests include image processing, pattern recognition, artificial neural networks, fuzzy logic, and visual media technology. He is a member of the International Pattern Recognition Society, European Neural Network Society, International Association of Science and Technology for Development, Swedish Society of Learning Systems, and a member of the IEEE.

\section{Efektyvi procedūra vizualiems defektams aptikti drožliu plokščiu paviršiuje}

\section{Jonas GUZAITIS, Antanas VERIKAS}

Šio darbo tikslas vaizdu analize ir automatiniu klasifikavimu grista paprasta ir efektyvi procedūra vizualiems defektams aptikti drožliu plokščiu paviršiuje. Vaizdo intensyvumo verčiu tikimybinis pasiskirstymas bei vaizdo dvimatès diskrečios Uolšo transformacijos koeficintai tai du automatinejje analizejje naudojami informacijos šaltiniai, pasitelkiant kuriuos išskiriami globalūs paviršiaus tekstūrą charakterizuojantys požymiai. Defektai aptinkami klasifikuojant šiu požymiu vektoriu i dvi klases: defektinis, kokybiškas. Klasifikatorius ne tik atlieka minètą sprendimą, bet ir ivertina šio sprendimo patikimumą. Atliekant eksperimentus su 200 vaizdų imtimi, visi šios imties vaizdai buvo klasifikuoti teisingai. 